\title{
Microstructural Evolution in NF616 (P92) and Fe-9Cr-0.1C-Model Alloy under Heavy Ion Irradiation
}

\author{
Cem Topbasi ${ }^{\mathrm{a}, *}$, Djamel Kaoumi ${ }^{\mathrm{b}}$, Arthur T. Motta ${ }^{\mathrm{a}, \mathrm{c}}$, Mark. A. Kirk ${ }^{\mathrm{d}}$ \\ ${ }^{a}$ Department of Materials Science and Engineering, Pennsylvania State University, University \\ Park, PA, 16802 USA \\ ${ }^{b}$ Department of Mechanical and Nuclear Engineering, University of South Carolina, 300 Main \\ St., Columbia, SC 29208, USA \\ ${ }^{c}$ Department of Mechanical and Nuclear Engineering, Pennsylvania State University, University \\ Park, PA, 16802, USA \\ ${ }^{d}$ Materials Science Division, Argonne National Laboratory, Argonne, IL, 60439, USA \\ * Corresponding author. Tel.: +1 814865 9709; fax: +1 8148658499 . \\ E-mail address:cempsu@gmail.com (C. Topbasi).
}

\begin{abstract}
In this comparative study, in situ investigations of the microstructure evolution in a $\mathrm{Fe}-9 \mathrm{Cr}$ ferritic-

martensitic steel, NF616, and a Fe-9Cr-0.1C-model alloy with a similar ferritic-martensitic microstructure have been performed. NF616 and Fe-9Cr-0.1C-model alloy were irradiated to high doses (up to 10 dpa) with $1 \mathrm{MeV} \mathrm{Kr}$ ions between 50 and $673 \mathrm{~K}$. Defect cluster density increased with dose and saturated in both alloys. The average size of defect clusters in NF616 was constant between 50 and $573 \mathrm{~K}$, on the other hand average defect size increased with dose in Fe-9Cr-0.1C-model alloy around $\sim 1 \mathrm{dpa}$. At low temperatures (50-298 K), alignment of small defect clusters resulted in the formation of extensive defects in Fe-9Cr-0.1C-model alloy around 2-3 dpa, while similar large defects in NF616 started to form at a high temperature of $673 \mathrm{~K}$ around $\sim 5 \mathrm{dpa}$. Interaction of defect clusters with the lath boundaries were found to be much more noticeable in $\mathrm{Fe}-9 \mathrm{Cr}$ 0.1C-model alloy. Differences in the microstructural evolution of NF616 and Fe-9Cr-0.1Cmodel alloy are explained by means of the defect cluster trapping by solute atoms which depends on the solute atom concentrations in the alloys.

Keywords: Generation-IV Nuclear Reactors, Sodium-Cooled Fast Reactor, Heavy Ion Irradiation, In Situ Technique, Transmission Electron Microscopy, Ferritic-Martensitic Alloys, Model Alloys.
\end{abstract}

\section{Introduction}

High chromium (9-12 wt.\%) ferritic-martensitic (F-M) steels are amongst the candidate in-core structural materials for future fusion energy systems and one of the Generation IV (GEN-IV) fission energy systems, the Sodium Fast Reactor (SFR) [1]. F-M steels are considered for fuel 
cladding and internal applications in SFR, as well as first wall and blanket materials in future fusion concepts due to their high resistance to irradiation induced void swelling, good microstructural stability and good thermal properties [2]. For these applications the F-M steels are envisioned to operate at high temperatures (573-873 K) and have to withstand high levels of radiation damage (150-200 dpa) [1].

Normalized and tempered 9-12 wt.\% F-M steels typically exhibit the tempered martensite structure characterized by fine laths and sub-grains with a high dislocation density [2]. Major improvements in the high temperature creep resistance of 9-12 wt.\% Cr F-M steels were established through alloying in the last century [2]. The modifications in the composition of the F-M steels include fine adjustments in solid solution and precipitation strengthening elements, the combination of which resulted in significant improvements in material properties outside irradiation [2].

The high-energy neutrons produced during fusion and fission cause displacement damage. A distribution of interstitial and vacancy defect clusters of various sizes forms as a result of high energy displacement cascades [3, 4]. The fate of these defects plays a determining role in radiation effects such as hardening, embrittlement, growth, creep and swelling. The mechanisms of radiation damage and irradiation-induced microstructural evolution have been extensively studied in pure $\mathrm{Fe}$ and binary $\mathrm{Fe}-\mathrm{Cr}$ alloys [4-7]. Jenkins et al. reported results of both in situ and ex situ irradiations of pure Fe and Fe-(5-18)Cr alloys with 100-150 keV Fe and Xe ions at $298 \mathrm{~K}$, $573 \mathrm{~K}$ and $773 \mathrm{~K}$ [6-8]. Ultra-high purity $\mathrm{Fe}$ and binary $\mathrm{Fe}-\mathrm{Cr}$ alloys exhibited a ferritic structure with large grains and a low density of pre-existing dislocations and precipitates. At early doses, the irradiation damage in these systems appeared as small $(2-4 \mathrm{~nm})$ defect clusters (assumed to be loops) [7] while at higher doses strings of defect clusters and resolvable loops were observed [8]. Defect analysis showed that a mixed population of dislocation loops with $1 / 2<111>$ and $<100>$ Burgers vectors existed at $298 \mathrm{~K}$ and $573 \mathrm{~K}$. However, only dislocation loops with <100> Burgers vectors were present at $773 \mathrm{~K}$. In addition, the fraction of $<100>$ loops were found to be higher in pure Fe compared to $\mathrm{Fe}-8 \mathrm{Cr}$ alloy [6-8]. The mobility of defects in $\mathrm{Fe}-8 \mathrm{Cr}$ alloy was significantly reduced compared to pure Fe, resulting in smaller defects even at higher doses [68].

Although irradiations of pure $\mathrm{Fe}$ and $\mathrm{Fe}-\mathrm{Cr}$ binary alloys provide significant insights on the microstructural evolution of bcc Fe, the actual F-M steels should be studied to understand the underlying reasons of their superior material properties (mechanical properties, radiation resistance, etc.) over those of pure $\mathrm{Fe}$ and binary $\mathrm{Fe}-\mathrm{Cr}$ alloys. On the other hand, few studies have been performed on the microstructural evolution of F-M steels under irradiation [9-11].

This study reports the results of the in situ heavy ion irradiation of a Fe-9Cr-0.1C model steel and a Fe-9Cr commercial F-M steel, NF616. NF616 (ASTM designation: P92) is a thirdgeneration F-M steel with a creep rupture strength of $132 \mathrm{MPa}\left(10^{5} \mathrm{hrs}\right)$ at $873 \mathrm{~K}$ [12] which makes it a promising candidate material for in-core applications in SFR and fusion energy systems. The ternary model alloy (referred as $9 \mathrm{Cr}$-model in the text) was designed to exhibit the typical microstructure of commercial F-M steels (in terms of lath martensitic structure and precipitates) without the confounding factor of additional alloying elements.

In situ irradiations of NF616 and 9Cr-model alloy were conducted with $1 \mathrm{MeV}$ Kr ions between $50 \mathrm{~K}$ and $673 \mathrm{~K}$ at the Intermediate Voltage Electron Microscope (IVEM) at Argonne National 
Laboratory. In situ heavy ion irradiations in TEM allow monitoring the development of microstructural changes (defects, voids, precipitates) in electropolished thin-foils under irradiation [13].

The objective of this paper is to present a comparison of microstructure evolution during in situ irradiations of NF616 and 9Cr-model alloy to contribute towards the understanding of the stability of the F-M structure under irradiation. Initial results of this study were published elsewhere $[9,14,15]$.

\section{Experimental details}

NF616 was provided by the Japan Atomic Energy Agency and the model alloy was fabricated at Ames Laboratory by arc melting of high purity starting elements. The chemical composition of NF616 and 9Cr-model alloy is given in Table 1. The model alloy has similar Fe, $\mathrm{Cr}$ and C content as NF616. NF616 was austenized at $1343 \mathrm{~K}$ for $1 \mathrm{~h}$, air cooled, then tempered at $1043 \mathrm{~K}$ for $2 \mathrm{~h}$ and air cooled. Similarly, model alloy was normalized at $1273 \mathrm{~K}$ for $1 \mathrm{~h}$, air cooled, then tempered at $1023 \mathrm{~K}$ and air cooled.

The initial microstructures of NF616 and the 9Cr-model alloy were characterized using optical microscopy, scanning electron microscopy (SEM) and transmission electron microscopy (TEM). Electropolished thin-foils of NF616 and 9Cr-model alloy were irradiated in situ with $1 \mathrm{MeV} \mathrm{Kr}$ ions to $10 \mathrm{dpa}$ at temperatures between $50 \mathrm{~K}$ and $673 \mathrm{~K}$ at the IVEM-TANDEM Facility at the Argonne National Laboratory.

SRIM software [16] was used to calculate the ion beam-induced damage in NF616 and 9Crmodel alloy in terms of dpa.The damage production cross-section for the $1 \mathrm{MeV} \mathrm{Kr}$ irradiation of NF616 is shown in Fig 1. The damage production estimated by SRIM varies weakly along the depth direction within the foil due to the high energy of $\mathrm{Kr}$ ions. Thus, depth-averaged values obtained from SRIM are used. The average number of displacements per ion per angstrom, is determined to be $\sim 2.4$ andthe dose rate was estimated to be $1.4 \times 10^{-3} \mathrm{dpa} / \mathrm{s}$ using the full cascade mode of the SRIM Monte Carlo code. The displacement energies $40 \mathrm{eV}$ for $\mathrm{Fe}$ and $\mathrm{Cr}$, and 28 $\mathrm{eV}$ for $\mathrm{C}$ [16], such that $1 \mathrm{dpa}$ corresponded to a fluence of $\sim 3.510^{18} \mathrm{ions} / \mathrm{m}^{2}$ in both alloys.

All observations were performed by recording two-beam bright field and dark field images by exciting 110-type reflections, with the TEM operated at an acceleration voltage of $200 \mathrm{kV}$. Irradiation was paused at certain doses to capture TEM images and to characterize the radiation damage. The sequential imaging of defect accumulation observed in a fixed location in the sample allowed accurate measurements of number density and size distribution of defects. Approximate foil thicknesses of the areas followed under irradiation are moderate $(\sim 100 \mathrm{~nm})$, as determined by post-irradiation EELS measurements. Therefore, minor differences in thickness of these areas are not expected to significantly affect areal density values. The definite difference in thickness of these areas could not be quantified because of the difficulty of finding the specific areas after irradiation. In addition video recording was performed with a coupled camera during irradiation for subsequent frame-by-frame analysis (time resolution of $34 \mathrm{~ms}$ ). The samples were electropolished by using a $5 \% \mathrm{HClO}_{4}$ and $95 \% \mathrm{CH}_{3} \mathrm{OH}$ solution cooled down to $233 \mathrm{~K}$ by liquid nitrogen. Details of the sample preparation and of the in situ heavy-ion irradiation experiment were published elsewhere $[9,14,15]$. 
Table 1. Composition of NF616 and model alloy in wt.\%.

\begin{tabular}{cccccccccccccc}
\hline Alloy & $\mathrm{Fe}$ & $\mathrm{C}$ & $\mathrm{Cr}$ & $\mathrm{Si}$ & $\mathrm{P}$ & $\mathrm{S}$ & $\mathrm{V}$ & $\mathrm{Mn}$ & $\mathrm{Ni}$ & $\mathrm{Nb}$ & $\mathrm{Mo}$ & $\mathrm{W}$ & $\mathrm{Al}$ \\
\hline NF616 & Bal. & 0.1 & 8.8 & 0.1 & 0.01 & 0.003 & 0.2 & 0.5 & 0.2 & 0.06 & 0.5 & 1.9 & 0.005 \\
Model & Bal. & 0.07 & 8.7 & - & $<0.005$ & 0.001 & - & - & - & - & - & - & - \\
Alloy & & & & & & & & & & & &
\end{tabular}

\section{Results}

\subsection{Microstructure of NF616 and 9Cr-model alloy}

The as-received microstructures of NF616 and 9Cr-model alloy are shown in Fig. 1. Figs.1(a) and 1(b) show optical microscopy images of the microstructure observed in the two materials. Because NF616 and 9Cr-model are positioned within the fully martensitic domain of the Schaeffler-Schneidner Diagram [17]. Figs.1(c) and 1(d) display secondary electron SEM images of the lath structures inside the large prior austenite grains in the two materials. A high density of $\mathrm{M}_{23} \mathrm{C}_{6}$ and $\mathrm{M}(\mathrm{C}, \mathrm{N})$ precipitates (where $\mathrm{M}$ denotes a metallic element) are present along lath boundaries and prior austenite boundaries in NF616 [18]. The density of precipitates is lower in the 9Cr-model alloy due to the lower concentration of carbide stabilizers in this alloy. Figs.1(e) and 1(f) show TEM images of both materials showing similar elongated lath structure observed in NF616 and 9Cr-model alloy. In NF616 a high density of pre-existing dislocations formed during the martensitic transformation in the NF616 that are inhomogeneously distributed within the laths is observed. NF616 exhibits the tempered martensite structure typically observed in 9$12 \mathrm{Cr}$ F-M steels. As shown in Fig. 1, the microstructure of the 9Cr-model alloy replicates the F$\mathrm{M}$ structure (lath/subgrain and prior-austenite grain boundaries) of NF616 reasonably well.

\subsection{Irradiation induced microstructure evolution}

Figure. 2 shows a series of two-beam dark field TEM images taken with the 110 reflection of specific areas in NF616 and 9Cr-model alloy which were followed during irradiations conducted at $50 \mathrm{~K}$. Fig.3 shows similar series taken during irradiations at $473 \mathrm{~K}$. The microstructural evolutions of NF616 and 9Cr-model alloy at $50 \mathrm{~K}$ showed are quite similar at low doses. Both alloys exhibit a threshold dose, around 0.1-0.3 dpa in this temperature regime, below which no defects are seen and above which small defect clusters become visible. Once formed these defect clusters did not grow until the end of irradiation (8.2 dpa in NF616 and and 4 dpa in the 9Crmodel alloy). The defect clusters are homogeneously distributed and the cluster density increases until reaching saturation. Rather than a static defect population, the in-situ observations showed that defects were constantly created and eliminated throughout the irradiation, while the overall density was constant. Although thermal migration of defects may not occur at $50 \mathrm{~K}$, defect motion can still be induced either by direct cascade impact or by pre-existing far field strain fields [19]. Indeed a significant fraction of defect clusters in NF616 and 9Cr-model alloy exhibited sudden jumps over $<10 \mathrm{~nm}$ under the ion beam. These sudden jumps were observed to occur in a time shorter than the time between subsequent video captured frames (34 ms or less) that the energy transferred from the displacement cascade was responsible for defect motion at these temperatures. 
The facts that defect motion and appearance/disappearance is only observed when the ion beam is hitting the sample (no defect motion under electron beam alone) and visible defect jumps occur in a specific direction (consistent with glide along the dislocation loop glide cylinder) point out a cascade-governed defect motion process. In addition molecular dynamics simulations suggest that defect trapping solutes occurs in the material [20,21] (and references therein). According to the suggested mechanism, energy transferred from cascades causes de-trapping of solute trapped defect clusters leading to allowed glide in their glide cylinder till they are trapped again by another solute atom or complex.

A.A. Kohnert et al used clusters dynamics models to describe formation and evolution of 2-5nm defect clusters in ferritic alloys under irradiation. The cluster dynamics approach involves integrating a coupled set of reaction-diffusion equations, and it can be considered as a modification of the classical rate theory model which aims to quantify the irradiation-induced microstructural evolution $[20,21]$.

To quote A.A. Kohnert et al [20]: "In such a trap mediated diffusion environment, the ultimate mobility of loops is governed by a series of ballistic detrapping events induced by energetic recoils. Following each of these events, a crowdion bundle glides with low activation energies until encountering a new trapping site. The length and frequency of these apparent hops are determined by the trap density and irradiation condition, respectively."

A.A. Kohnert et al added a heavy ion beam-assisted mobility term (second term in Eq.1) to account for the defect cluster jumps constantly observed during in situ TEM irradiations, where the first term in Eq.1 describes the standard Arrhenius type diffusion. is a diffusion prefactor and is the migration energy for the cluster, $T$ is the temperature and $k$ is the Boltzmann constant [20, 21].

Second term formulates the frequent discrete motion of thermally immobile (possibly trapped) defect clusters under irradiation which can be attributed to the ballistic de-trapping events created by energetic recoils. In the second term, is the activation frequency, is the discrete hop length (distance between jumps governed by the density of impurity traps), and is the dimensionality of diffusion[20,21].

A.A. Kohnert et al calculated the saturation density of visible defect clusters for $1 \mathrm{MeV} \mathrm{Kr}$ ion irradiation of ferritic-martensitic alloys (NF616 and a Fe-12Cr-0.1C model alloy) with beam activated diffusion applied in the form of Eq.1. Results were in good qualitative agreement with the experimental data $[20,21]$.

Fig.3 illustrates the evolution of the irradiation induced microstructures in NF616 and 9Cr-model alloy when irradiated at $473 \mathrm{~K}$. At this irradiation temperature, although interstitials and vacancies are expected to be thermally mobile in pure Fe vacancy clusters should still not be thermally mobile [19]. For the alloys studied, the microstructure development in NF616 under irradiation at $50 \mathrm{~K}$ and $473 \mathrm{~K}$ was quite similar. After a threshold dose, the defect cluster density started to increase until saturation with no apparent change in the size of defect clusters. In contrast, the diameter of defect clusters in the 9Cr-model alloy started to increase around $1 \mathrm{dpa}$ likely by a combination of absorption of point defects into clusters. Resolvable loops in the $9 \mathrm{Cr}-$ 
model alloy started to become visible around 2-3 dpa and interaction of these loops (at this temperature) resulted in the formation of dislocation tangles at higher doses, as shown in Figs. 3.(g-1).

Figs. 4(a) and (b) show dark field images of grain boundaries separating neighboring laths in NF616 and 9Cr-model alloy irradiated to doses of 9-10 dpa at $573 \mathrm{~K}$ and $473 \mathrm{~K}$, respectively. These two-beam dark field images of the laths oriented close to the (111) zone axis were taken using 110-type g vectors to allow direct comparison between the images. While in NF616 irradiation-induced microstructural evolution at $573 \mathrm{~K}$ mirrored that observed at $50 \mathrm{~K}$ and $473 \mathrm{~K}$, a stronger effect of irradiation temperature is seen in 9Cr-model alloy. In particular no apparent interaction was observed between the irradiation-induced defects with the preexisting microstructure, as illustrated by the unchanged grain boundary in NF616 (Fig. 4 (a)). On the other hand, in the 9Cr-model alloy irradiated at 473K, denuded zones appeared in some laths as shown in Fig. 4(b), suggesting that mobile defect clusters in the vicinity of the lath boundaries can be absorbed at the lath boundaries. In the interior of the grains (away from the lath boundaries), microstructure development showed extended loops and loop entanglement at 10 dpa.

Fig. 5 (a) shows representative high-dose defect structures observed in NF616 after irradiation to $7.4 \mathrm{dpa}$ at $673 \mathrm{~K}$. The gradual coalescence of defect clusters in NF616 at $673 \mathrm{~K}$ resulted in the formation of the vertically aligned defects as shown by the arrows in Fig. 5(a). Similarly aligned finger-shaped defects were reported to form in binary in pure $\mathrm{Fe}$ and $\mathrm{Fe}-8 \% \mathrm{Cr}$ alloys under heavy ion irradiation at $773 \mathrm{~K}$. Jenkins et al characterized them as two families of edge-on $<100>$ loops [6].

These are compared to microstructures seen in 9Cr-model alloy after irradiation to $10 \mathrm{dpa}$ at $298 \mathrm{~K}$ (fig. 5(b)) and after irradiation to $10 \mathrm{dpa}$ at $180 \mathrm{~K}$ (fig. 5(c)). Similar extended defect structures are observed which are approximately aligned with the $<110\rangle$ directions. This phenomenon of self-ordering of defect in the 9Cr-model alloy was described and discussed in details in [15].These defect strings developed further into complex rafts linking together defect segments, especially in the thicker parts of the sample. M. Hernandez- Mayoral et al reported formation of similar defect strings with $1 / 2<111>$ Burgers vectors [8]. These self-ordered structures are thought to result from elastic interactions between defect clusters in the foil when their density is high enough and the resultant internal strains may be the main reason for the development of the aligned structure [14]. The strain caused by a high density of loops would be minimized by the regular arrangement of defects clusters. The preferred crystallographic orientation of defect arrays may be driven by the minimization of elastic interaction energy between defect clusters.

The defect cluster density was measured as a function of dose for all temperatures and defect density values were normalized to the highest value acquired for each alloy, plus the error bar. Fig. 6. shows the normalized defect cluster density with increasing dose for NF616 during irradiation at $50 \mathrm{~K}, 473 \mathrm{~K}$ and $673 \mathrm{~K}$ to $8 \mathrm{dpa}$ and of the defect cluster density ion the $9 \mathrm{Cr}$-model alloy when irradiated at $50 \mathrm{~K}$ and $473 \mathrm{~K}$ to $2 \mathrm{dpa}$. The measured defect cluster density in NF616 at $~ 6 \mathrm{dpa}$, while that for the 9Cr-model alloy saturates earlier in dose. The defect cluster density in both alloys decreased with increasing temperature. Between $50 \mathrm{~K}$ (highest density) and $473 \mathrm{~K}$ the decrease in saturation density of NF616 was $~ 10 \%$ while for the 9Cr-model alloys showed a decrease of $\sim 70 \%$, in its saturation defect cluster density. In NF616 the irradiation at $673 \mathrm{~K}$ 
shows a large decrease in defect cluster density, suggesting the onset of a thermally driven regime at that temperature. This onset of thermally driven regime was seen already at $473 \mathrm{~K}$ for the 9Cr0-model alloy.

Fig. 7 (a) shows the average defect cluster size in NF616 at $50 \mathrm{~K}, 473 \mathrm{~K}$ and $673 \mathrm{~K}$. It is clear that the defect size does not change with either dose or temperature, except at $673 \mathrm{~K}$. For the $9 \mathrm{Cr}$-model alloy the defect size is approximately constant at these low doses for irradiations at $50 \mathrm{~K}$ and $473 \mathrm{~K}$. The average defect size in NF616 was around 3-4 $\mathrm{nm}$ at $50 \mathrm{~K}$ and $473 \mathrm{~K}$, while it almost doubled at $673 \mathrm{~K}$. On the other hand, the average defect size was $35-38 \%$ larger in the material irradiated at $473 \mathrm{~K}$ than that measured at $50 \mathrm{~K}$ in the $9 \mathrm{Cr}$-model alloy.

There was no evidence of void formation or precipitation in either alloy at the irradiation doses and temperatures studied.

\section{Discussion}

The common characteristics and dissimilarities between the irradiation induced microstructural evolution in NF616 and in 9Cr-model alloy are as follows:

(i) Defect formation and visibility: The initial visible damage (2-4 nm sized white dots in DF TEM images) under the $1 \mathrm{MeV} \mathrm{Kr}$ irradiations appeared at cascade overlap doses. This indicates formation of defect clusters in cascade events by the impact of cascades on a volume which contains a background density of defects created by prior ion collisions that are not visible in TEM (i.e. under TEM resolution). This is in agreement with previous studies of the onset of defect accumulation in bcc Fe under ion irradiation. The collapse of individual, isolated cascades to visible defects in Fe did not occur under heavy-ion irradiations however visible damage was observed at relatively high irradiation doses $[7,11$, 22]. At cryogenic temperatures $(50 \mathrm{~K})$ irradiation-induced defect clusters do not become visible until higher doses. This suggests that TEM-invisible defects become visible/detectable only when they have grown to a size larger than the TEM resolution limit, by cascade induced defect cluster motion or direct overlap. It was not possible to determine the nature of the nanometer sized defects observed in our in situ irradiations since size of the defect clusters is too small to apply inside-outside contrast method. Nevertheless, previous studies about the irradiation-induced defects in Fe showed that defects that were large enough to characterize were highly mobile interstitials. On the other hand, relatively immobile vacancy clusters remain the microstructure as sub-visible $(<2 \mathrm{~nm})$ defect clusters that are not observable by TEM. The interstitial clusters are likely created directly in cascades and trapped by impurities in the material.

(ii) Defect motion/jumps: The defect clusters constantly appeared and disappeared under theion beam. Flickering and sudden jumps of white dots in DF TEM images were observedearly on during the irradiations and continued above the saturation dose resulting in a dynamic picture under irradiation. The one-dimensional jumps of clusters were observed in both alloys. However, "to-and-fro hops" of clusters (i.e. sudden back and forth (1D) movements about the same position) often observed in ultra-high purity iron systems [8] were more rarely seen in these more complex alloys and the defect jumps had a smaller rattling frequency; the jump 
distances in the current alloys were less than the jumps observed by others in pure iron [8]. When such cluster hops occurred during irradiation, the cluster could spend a few seconds before jumping back to the previous position, suggesting that impurity trapping slows down the motion In addition mobile interstitial clusters may interact with other interstitial and vacancy clusters during their glide between traps (solute atoms and complexes). These interactions can change the size of the interstitial clusters (increase in the case of interstitial and decrease in the case of a vacancy). The effect of these coalescence events on the mobility of interstitial clusters is unknown and requires further investigation.

(iii) Defect density dependence on dose: The areal density of the TEM-visible defect clusters increased with dose, approaching an apparent dynamic saturation, in which defects are constantly created and destroyed at the same rate. It is expected that, with increasing irradiation dose, the vacancy cluster concentration will steadily increase such that interstitial clusters will increasingly gather vacancies as they glide between solute traps. This will cause saturation in defect density and limit the interstitial cluster size. In this scenario, a steady state could be established in which a stable concentration of interstitial clusters of fixed size is formed which will coexist with a high density of vacancies which will preclude further growth.

(iv) Defect size: The constant defect size in NF616 between $50 \mathrm{~K}$ and $573 \mathrm{~K}$ indicates a significant reduction in the mobility of defects in this wide temperature range, likely caused by impurity trapping. On the other hand, larger defects form in the $9 \mathrm{Cr}$-model alloy at $473 \mathrm{~K}$. In addition the decrease in the density of defects between $50 \mathrm{~K}$ and $473 \mathrm{~K}$ was more pronounced in the 9Cr-model alloy compared to NF616. This can be attributed to the higher mobility of defects in the model alloy which can result in a higher rate of recombination and defect loss to the sinks. In accordance, defect-denuded zones along the grain boundaries were observed only in the $9 \mathrm{Cr}$ model alloy, indicating enhanced transport of defects to sinks in the model alloy relative to NF616 whose chemical composition indicates higher solute content.

The exact mechanisms of loop growth are expected to affect the dose dependence of the average loop size (as well as the loop density), i.e. whether loop growth happens by (i) diffusion and absorption of single point defects, (ii) by diffusion and absorption of smaller clusters (iii) by cascade overlap, or (iv) by loop coalescence. In contrast with what is seen in pure Fe, loop coalescence was not observed in either alloy likely because of impurity trapping; however small black dots were seen decorating larger loops in 9Cr-model alloy suggesting that the larger loops can grow by absorption of smaller clusters and point defects. These can be small clusters formed in the direct vicinity of the loops, either swept there by cascade impact at the lowest temperatures or by thermal migration at the highest temperatures above stage II.

The differences in the microstructural evolution of NF616 and 9Cr-model alloy including the formation of denuded zones, the effect of temperature on average defect size and defect density could be explained within the framework of the trapping of defects by solute atoms due to the difference in solute concentration between the two alloys. Molecular dynamics simulations [3, $23,24]$ showed that interstitial and substitutional point defects and their clusters can form directly in cascades and interstitial defect clusters $(<100$ member $)$ diffuse with activation energies comparable to one of single self-interstitials in the absence of solute atoms by exhibiting a collective motion of interstitial crowdions [25].The distribution of solute atoms in NF616 and 9Cr-model alloy and the magnitude of the binding energy between solute atoms an defects may 
strongly affect their mobility therefore controlling processes such as defect coalescence and migration to sinks.

The concentration of alloying elements in NF616 is considerably higher than in the 9Cr-model alloy. Although a fraction of these solute atoms precipitate as carbides and other particles at lath and prior austenite boundaries in NF616 during the tempering treatment, a fraction of these alloying elements remain in the matrix and may form Cottrell atmospheres that can pin dislocations and can reduce the mobility of defect clusters[2, 26]. Arakawa et al reported onedimensional thermal motion of isolated interstitial defect clusters $(6-20 \mathrm{~nm})$ towards $<111>$ direction in pure Fe, in the absence of stress [27]. The immobilization of defects below $450 \mathrm{~K}$ in samples with $0.8 \mathrm{ppm} \mathrm{C}$ was attributed to the formation of the Cottrell atmosphere around the defects by the authors [27]. The relatively low density of $\mathrm{C}$ and $\mathrm{N}(\sim 0.8 \mathrm{ppm})$ in Fe necessitated these slow moving interstitials to diffuse towards the defect clusters (or vice versa) to immobilize them. In addition substitutional solute atoms can act as barriers to defect motion by creating strain fields in the matrix, depending on their misfit factor [28]. In situ electron irradiations conducted by T. Hamaoka et al showed a significant decrease in the mobility of defect clusters in $\mathrm{Fe}$ upon addition of 50 appm oversized $\mathrm{Cu}$ and undersized $\mathrm{Si}$ [28].

A high density of solute atoms in the matrix of NF616 can trap irradiation induced point defects once they are created. This is in agreement with our observations of no temperature dependence of microstructure evolution in NF616 up to $673 \mathrm{~K}$ in contrast with the 9Cr-model alloy for which a temperature effect was noticed above $180 \mathrm{~K}$. A higher density of matrix solute atoms can also explain the shorter length of ion beam induced sudden jumps in NF616 than in 9Crmodel alloy. The ion beam induced sudden jumps in 9Cr-model alloy were in turn found to be shorter than those seen during irradiation of pure Fe [8]. That is, as the solute concentration increases, the ion beam induced jumps become shorter.

Results indicate a wide non-thermally controlled temperature regime $(50 \mathrm{~K}<\mathrm{T}<573 \mathrm{~K})$ in NF616 which is characterized by constant defect size and restricted defect motion. On the other hand, this "cascade-governed regime" in 9Cr-model alloy seems to be limited to lower temperatures $(\mathrm{T}<298 \mathrm{~K})$. Dislocation loop growth and formation of extended defect structures involving resolvable loops could be observed in the $9 \mathrm{Cr}$-model alloy at $473 \mathrm{~K}$ and $573 \mathrm{~K}$, whereas these were only seen in NF616 at $673 \mathrm{~K}$ and to a lesser degree. We note however that the gradual coalescence of defects into extended defects was only observed under the ion beam (i.e. not under thermal annealing) which indicates that irradiation enhanced thermal diffusion or cascade driven diffusion is necessary at $673 \mathrm{~K}$.

(v) The alignment of defects: Rafts of defects formed aligned along $<110>$ directions at high doses in 9Cr-model alloy between $50 \mathrm{~K}$ and $573 \mathrm{~K}$ (Fig. 5). These extended defects can form as a result of a high density of evenly distributed defect clusters in $9 \mathrm{Cr}$-model alloys which can result in short-range elastic interactions between defect clusters in close vicinity to each other causing them to align and thus minimize strain energy. Kinetic Monte Carlo simulations conducted by M. Wen et al suggest that mutual strain fields between interstitial clusters can cause them to move in their glide cylinder [29]. There was less indication of defect alignment in NF616 likely because of solute pinning.

\section{Conclusions}


In situ irradiations of NF616 and 9Cr-model alloy were performed with $1 \mathrm{MeV}$ Kr ions between $50 \mathrm{~K}$ and $673 \mathrm{~K}$ to investigate microstructure evolution in these alloys using identical sample preparation and in situ irradiation setup to enable direct comparison. The main results are as follows:

1. Quantitative analysis indicates that defect cluster density increases with dose and saturates in both alloys during irradiations conducted between 50 and $673 \mathrm{~K}$.

2. The average size of irradiation-induced defect clusters in NF616 does not vary with dose or temperature between 50 and $573 \mathrm{~K}$ whereas the average defect size increases moderately with dose in 9Cr-model alloy.

3. Extended defect structures resulting from the alignment of small defect clusters were observed at high doses in the 9Cr-model alloy for irradiations conducted between 50 and $298 \mathrm{~K}$ whereas larger defect structures started to form in NF616 only at $673 \mathrm{~K}$. These "self-ordered" defect structures were finer in scale and less aligned than in the $9 \mathrm{Cr}$-model alloy.

4. Interaction of the irradiation induced defects with the pre-existing lath boundaries was observed in the 9Cr-model alloy but not in NF616.

5. No voids or irradiation-induced precipitates were observed in either alloy for the irradiation temperatures and doses studied.

6. In general the results were consistent with increased defect cluster trapping by solutes in NF616 than in 9Cr-model alloy, leading to a wider temperature range where thermal effects were less significant in NF616, which emphasizes the impact of solutes on the evolution of irradiation 
induced microstructure even when the pre-existing (lath/precipitate) microstructure is similar.
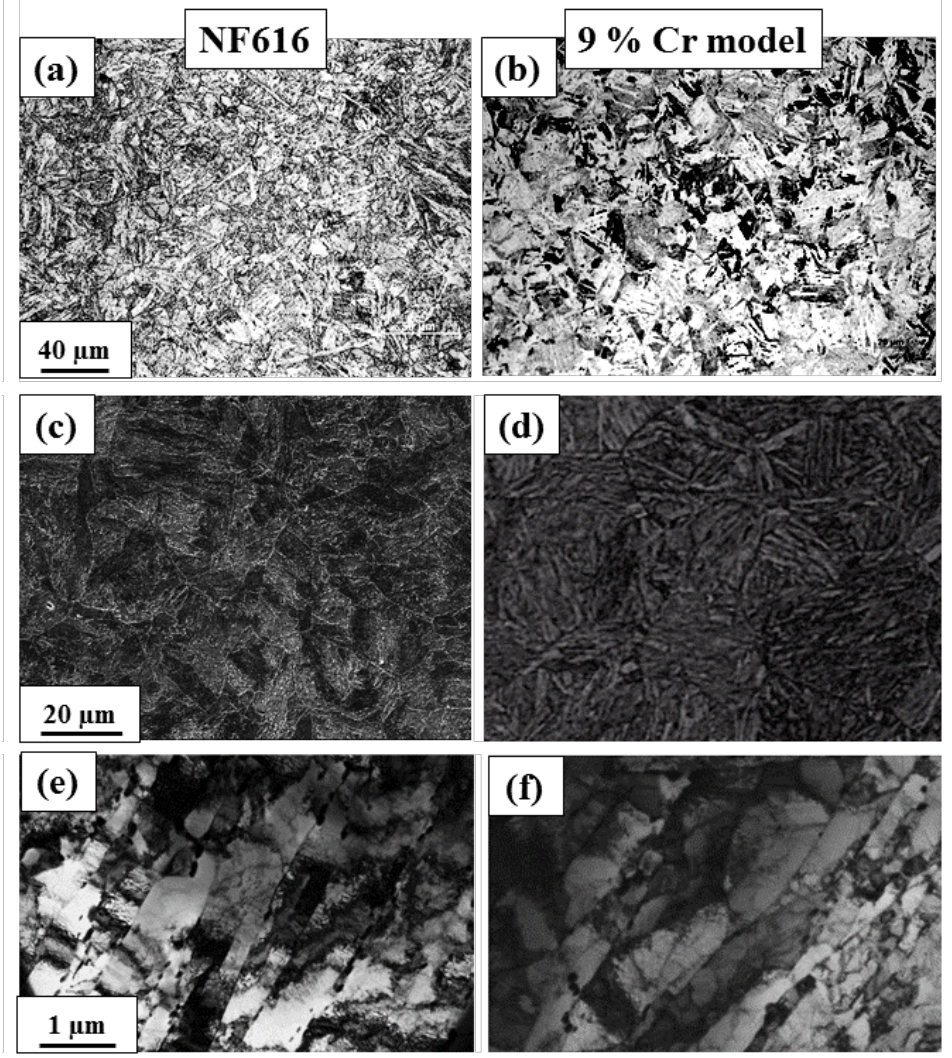

Fig. 1. (a,b) Optical microscopy images of NF616 and 9Cr-model alloy showing the tempered martensite microstructure. (c,d) SEM images of prior-austenite grains and the lath structure in the NF616 and 9Cr-model alloy. (e,f) Bright field TEM images of elongated lath structure inside prior-austenite grains of NF616 and 9Cr-model alloy.
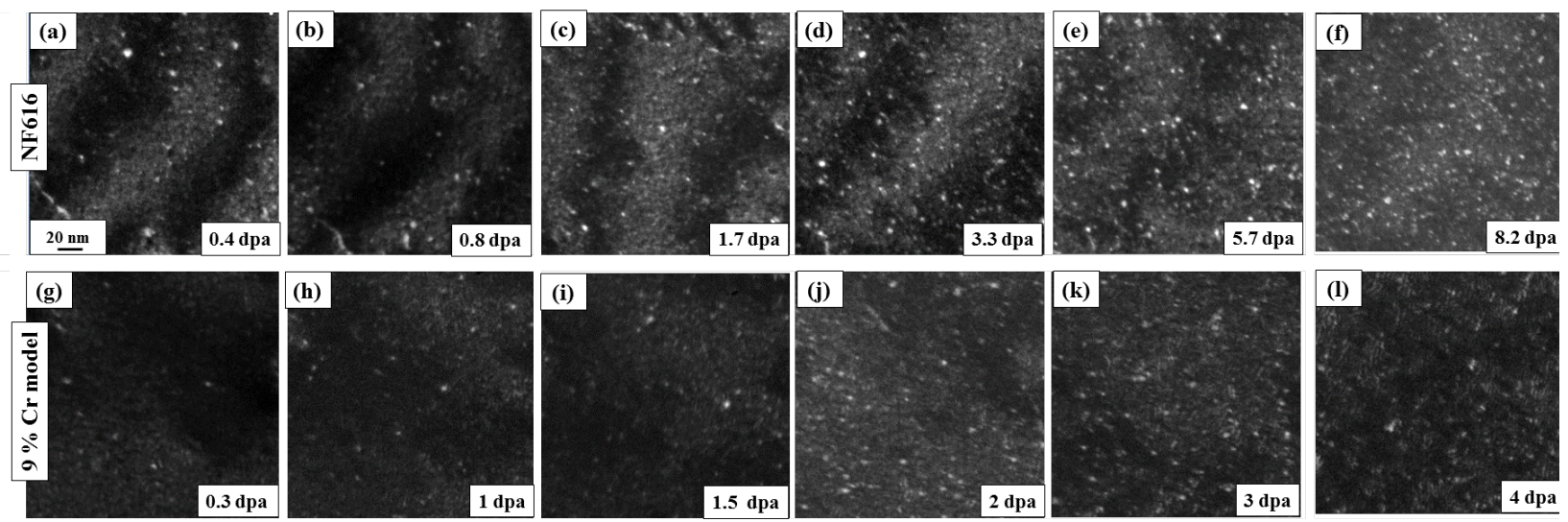

Fig. 2. Dark field transmission electron micrographs showing the microstructure evolution of NF616 (a-f) and 9Cr-model alloy (g-l) under $1 \mathrm{MeV}$ Kr Irradiation at $50 \mathrm{~K}$. 

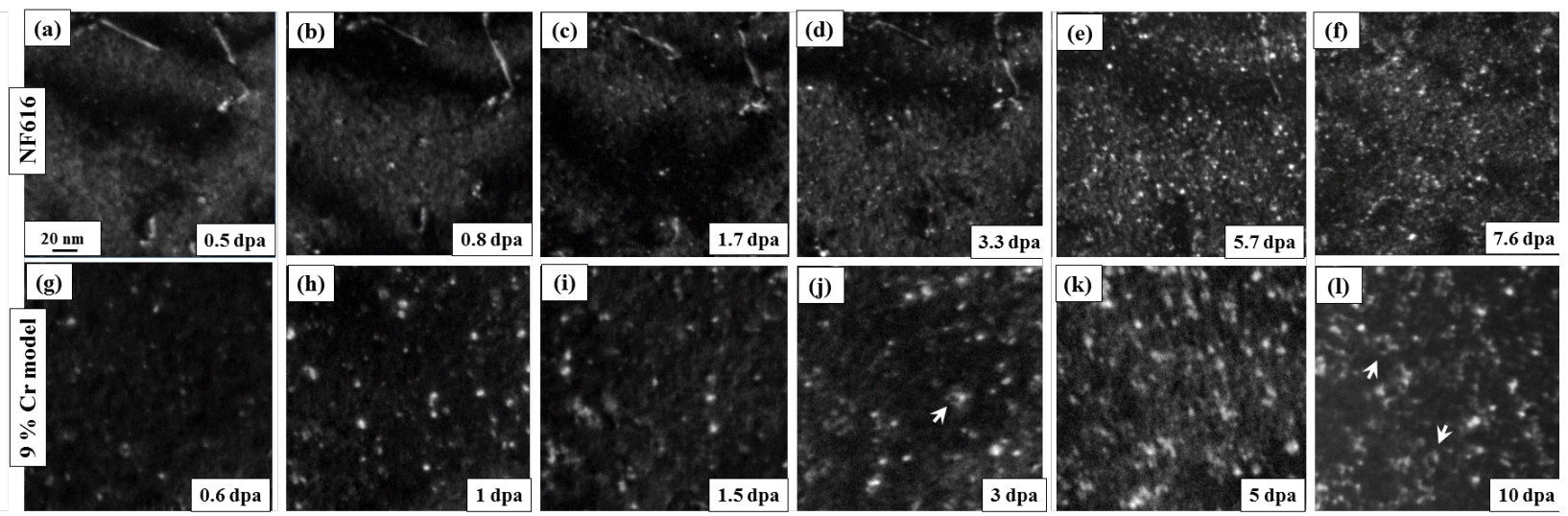

Fig. 3. Dark field transmission electron micrographs showing the microstructure evolution of NF616 (a-f) and 9Cr-model alloy (g-l) under $1 \mathrm{MeV}$ Kr Irradiation at $473 \mathrm{~K}$. Arrows indicate resolvable dislocation loops in the $9 \mathrm{Cr}$-model alloy.
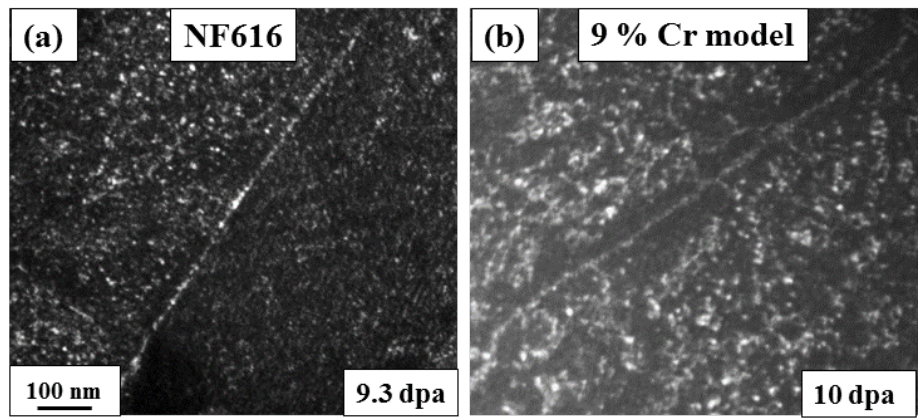

Fig. 4. Dark field transmission electron micrographs of the interaction of irradiation induced defects with the lath boundary in NF616 and 9Cr-model alloy under $1 \mathrm{MeV} \mathrm{Kr}$ Irradiation at 573 $\mathrm{K}$ and $473 \mathrm{~K}$, respectively.
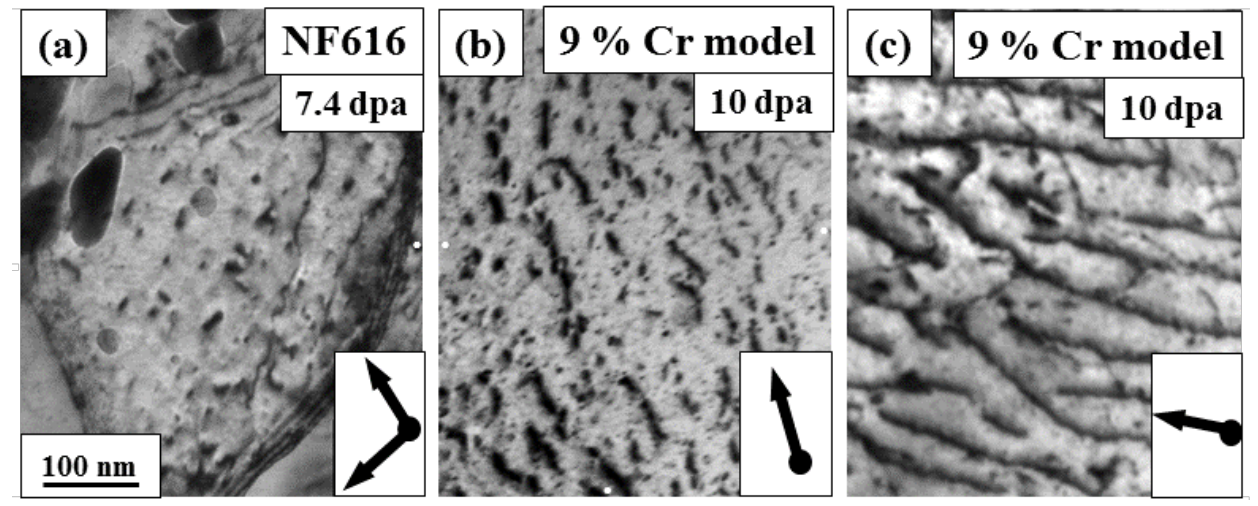

Fig. 5. Bright field transmission electron micrographs of extended defects observed in NF616 and $9 \mathrm{Cr}$-model alloy under $1 \mathrm{MeV} \mathrm{Kr}$ irradiations performed at $673 \mathrm{~K}$ (a), $298 \mathrm{~K}$ (b) and $180 \mathrm{~K}$ (c), respectively. Arrows indicate approximate directions of defect alignment in NF616 and 9Crmodel alloy. 

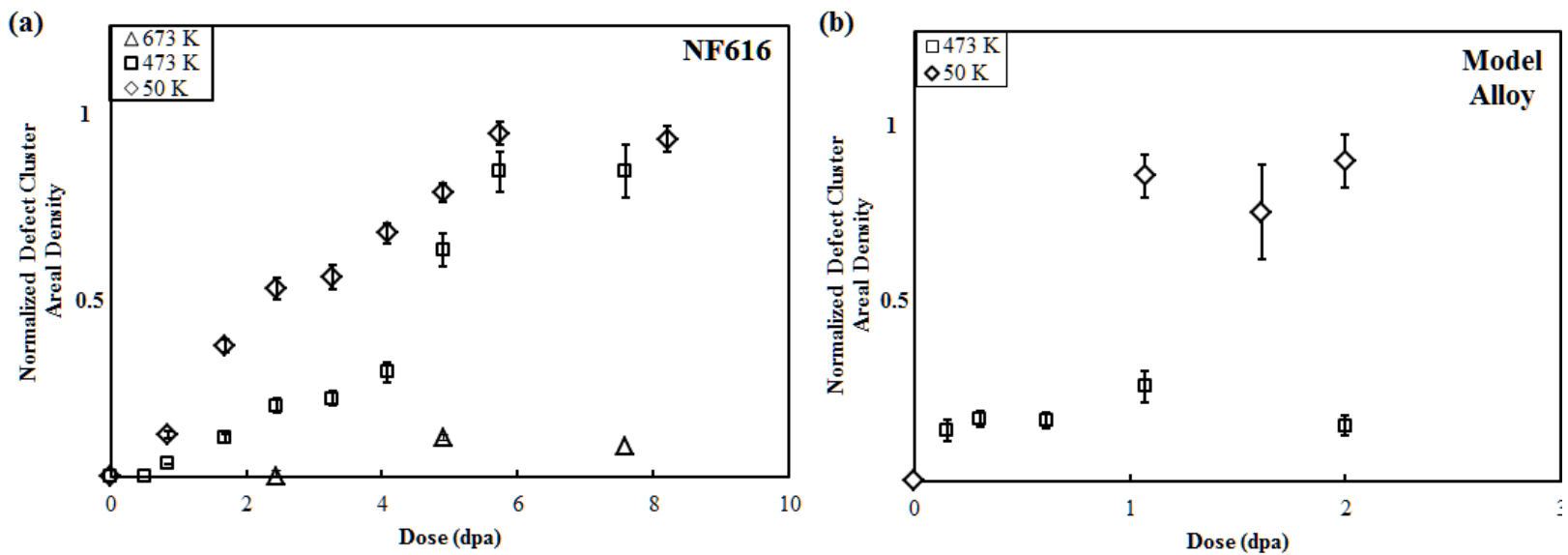

Fig. 6. Normalized defect cluster areal density of (a) NF616 and (b) 9Cr-model alloy as a function of dose at $50 \mathrm{~K}, 473 \mathrm{~K}$ and $673 \mathrm{~K}$. The defect cluster density is normalized to the highest value acquired at $50 \mathrm{~K}\left(\sim 0.005\right.$ defects.nm ${ }^{-2}$ at 5.5 dpa for NF616 and $\sim 0.006$ defects.nm $^{-}$ ${ }^{2}$ at 2 dpa for $9 \mathrm{Cr}-$ model alloy), plus the error bar.

(a)

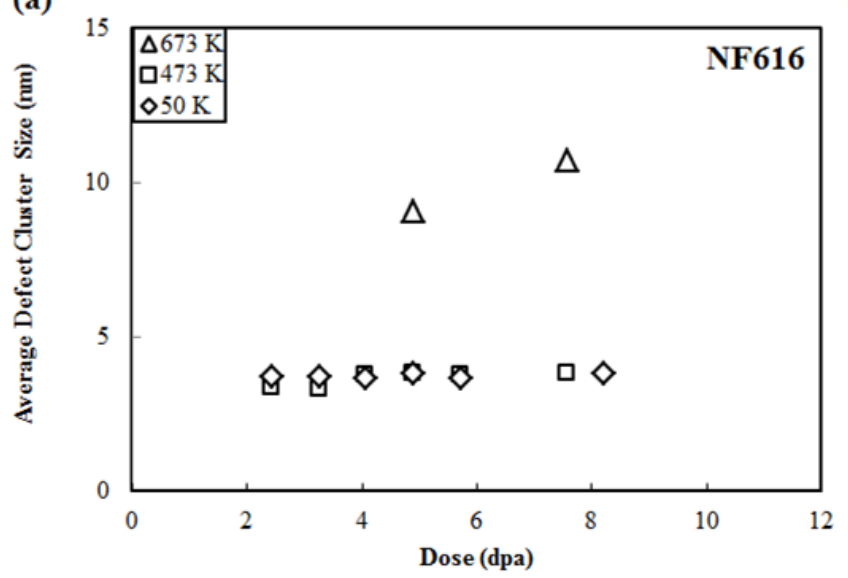

(b)

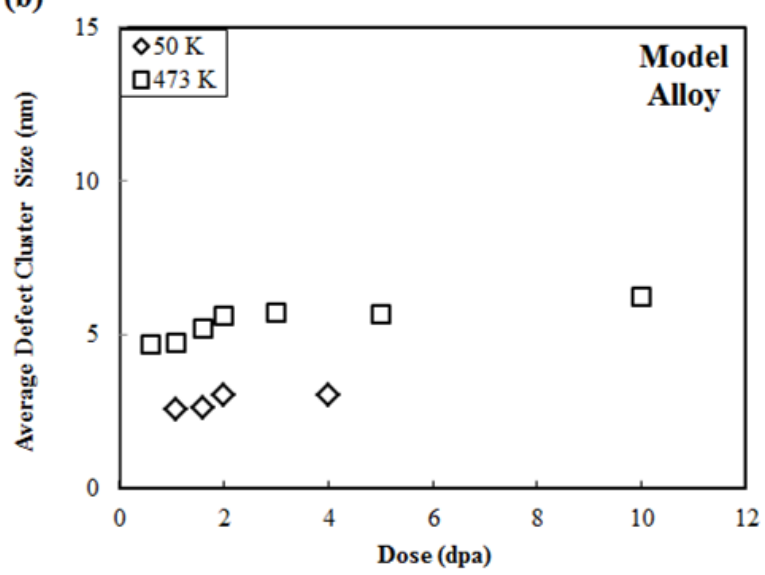

Fig. 7. (b) Average defect cluster size of (a) NF616 and (b) 9Cr-model alloy as a function of dose at $50 \mathrm{~K}, 473 \mathrm{~K}$ and $673 \mathrm{~K}$.

\section{Acknowledgements}

This work was funded by the Nuclear Energy University Programs (NEUP) Award number DEFG07-07ID14894 from the U.S. Department of Energy. The research conducted in the IVEMAccelerator facility at Argonne National Laboratory, which is supported as a User Facility by the 
U.S. Department of Energy, Basic Energy Sciences, under contract W-31-109-ENG-38. We thank Pete Baldo of Argonne National Lab for his help in performing the irradiation. We also thank Brian Wirth and Aaron Kohnert for helpful discussions.

\section{References}

[1] A Technology Roadmap for Generation IV Nuclear Energy Systems, US DOE NERAC and Generation IV International Forum, GIF-002-00, 2002.

[2] R.L. Klueh, D.R. Harries, "High Chromium Ferritic and Martensitic Steels for Nuclear Applications", American Society for Testing and Materials, West Conshohocken, PA, 2001.

[3] D.J Bacon, Y.N. Osetsky, R Stoller, R.E Voskoboinikov, J. Nucl. Mater. 323 (2003) 11.

[4] A.F. Calder, D.J. Bacon, A.V. Barashev, Y.N. Osetsky, Phil. Mag. Lett. 88 (2008) 43.

[5] M.R. Gilbert, Z. Yao, M.A. Kirk, M.L. Jenkins, S.L. Dudarev J. Nucl. Mater. 388 (2009) 36.

[6] M.L. Jenkins, Z. Yao, M. Hernández-Mayoral, M.A. Kirk, J. Nucl. Mater. 389 (2009) 197.

[7] Z. Yao, M. Hernández-Mayoral, M.L. Jenkins, M.A. Kirk, Philos. Mag. 88 (2008) 2851.

[8] M. Hernández-Mayoral, Z. Yao, M.L. Jenkins, M.A. Kirk, Philos. Mag. 88 (2008) 2881.

[9] C. Topbasi, A.T. Motta, M.A. Kirk, J. Nucl. Mater. 425 (2012) 48.

[10] T.R. Allen, L. Tan, J. Gan, G. Gupta, G.S. Was, E.A. Kenik, S. Shutthanandan, S. Thevuthasan, J. Nucl. Mater. 351 (2006) 174.

[11] H. Fukushima, Y. Shimomura, H. Yoshida, J. Nucl. Mater. 141-143 (1986) 938.

[12] D. Coutsouradis ,"Materials for Advanced Power Engineering Part 1", Proc. Int. Conf. Liège 1994, Dordrecht, Netherlands, 1994.

[13] M.A. Kirk, P.M. Baldo, A.C. Liu, E.A. Ryan, R.C. Birtcher, Z. Yao, S. Xu, M.L. Jenkins, M. Hernandez-Mayoral, D. Kaoumi, A.T. Motta, Microsc. Res. Tech. 72 (2009) 182.

[14] D. Kaoumi, J. Adamson, J. Nucl. Mater. 448 (2014) 233.

[15] D. Kaoumi, J. Adamson, M.A. Kirk, J. Nucl. Mater. 445 (2014) 12.

[16] J.F. Ziegler, SRIM-2008, v. 2008.40. <http://www.srim.org>.

[17] H. Schneider, Foundry Trade Journal,108 (1960) 562.

[18] P.J. Ennis, A. Zielinska-Lipiec, O. Wachter, A. Czyrska-Filemonowicz, Acta Mater. 45 (1997) 4901. 
[19] P. Ehrhart, P. Jung, H. Schultz, and H. Ullmaier, in Atomic Defects in Metals, edited by H. Ullmaier, Landolt-Börnstein, New Series, Group III, Vol. 25 (Springer-Verlag, Berlin, 1991)

[20] A.A. Kohnert, B.D. Wirth, Journal of Applied Physics 117 (2015) 154305.

[21] B.D. Wirth, X. Hu, A. Kohnert and D. Xu, Journal of Materials Research, 30(2015) 1440

[22] M. A. Kirk, M. Robertson, J. S. Vetrano, M. L. Jenkins, L. L. Funk, in: F. H. Garner, N. H. Packan, A. S. Kumar (Eds.), Radiation-Induced Changes in Microstructure: 13th International Symposium, ASTM STP 955, American Society for Testing and Materials, Philadelphia, PA, 1987, p. 48.

[23] A.F. Calder, D.J. Bacon, A.V. Barashev, Y.N. Osetsky, J. Nucl. Mater. 382 (2008) 91.

[24] B.D. Wirth, Science 318 (2007) 923.

[25] Y.N. Osetsky, D.J. Bacon, A. Serra, B.N. Singh, S.I. Golubov, Philos. Mag. 83 (2003)

61.

[26] A.H. Cottrell, B. Bilby, Proc. Phys. Soc. A, 62 (1949) 49.

[27] K. Arakawa, K. Ono, M. Isshiki, K. Mimura, M. Uchikoshi, H. Mori, Science 318 (2007) 956.

[28] T. Hamaoka, Y. Satoh, H. Matsui, J. Nucl. Mater. 433 (2013) 180.

[29] M. Wen, N.M. Ghoniem, B.N. Singh, Phil. Mag. 85 (2005) 2561. 